\title{
Estimulación cerebral profunda en enfermedad de Parkinson
}

\author{
Daniel Stiven Marin-Medina', Juan Felipe Quintero-Moreno' , Aníbal Valencia-Vásquez', Catalina Duque-Salazar², \\ Andrés Felipe Gil-Restrepo², Juan Pablo Castaño-Montoya², Daniela García-Rodríguez², Hans Carmona-Villada²
}

\section{RESUMEN}

Introducción: la enfermedad de Parkinson es considerada la segunda causa de enfermedad neurodegenerativa, en la que se destacan signos y síntomas motores como temblor, bradicinesia, rigidez e inestabilidad postural, acompañados de síntomas no motores como alteraciones del sueño, autonómicas, cognitivas, gastrointestinales, entre otras. El tratamiento farmacológico de la enfermedad al inicio suele ser útil, pero cuando los síntomas persisten, el tratamiento falla o no se toleran sus reacciones adversas, es necesario considerar alternativas como la estimulación cerebral profunda.

Metodología: revisión narrativa con énfasis en los aspectos clínicos de la terapia con estimulación cerebral profunda en los pacientes con enfermedad de Parkinson.

Discusión: la estimulación cerebral profunda es una técnica quirúrogica en la que se implantan electrodos en regiones cerebrales específicas, generalmente el núcleo subtalámico, globo pálido interno o núcleo ventral intermedio del tálamo, y se conectan a un marcapasos subcutáneo desde donde se modula eléctricamente la actividad de estas áreas. Esta terapia ha mostrado ser costo-efectiva, aporta beneficios considerables en la mejoría de los síntomas de la enfermedad de Parkinson y cuenta con evidencia clínica en los pacientes que han sido seleccionados correctamente.

\section{PALABRAS CLAVE}

Enfermedad de Parkinson; Neurocirugía; Terapia por Estimulación Eléctrica

1 Estudiante de pregrado de Medicina. Integrante del grupo de Investigación Applied Neuroscience, Universidad Tecnológica de Pereira, Colombia.

2 Médico integrante del grupo de Investigación Applied Neuroscience, Universidad Tecnológica de Pereira, Colombia.

Correspondencia: Daniel Stiven Marin Medina; cercaylejos@utp.edu.co

Recibido: octubre 02 de 2017

Aceptado: febrero 26 de 2018

Cómo citar: Marín-Medina DS, Quintero-Moreno JF, Valencia-Vásquez A, Duque-Salazar C, Gil-Restrepo AF, Castaño-Montoya JP, et al. Estimulación cerebral profunda en enfermedad de Parkinson. Iatreia. 2018 Jul-Sept;31(3): 262-273. D0I 10.17533/udea.iatreia.v31n3a04. 


\section{SUMMARY}

\section{Deep brain stimulation in Parkinson disease}

Introduction: Parkinson's disease is considered the second cause of neurodegenerative disease, in which motor signs and symptoms such as tremor, bradykinesia, rigidity and postural instability are highlighted, accompanied by non-motor symptoms such as sleep, autonomic, cognitive, gastrointestinal among others disturbances. The pharmacological treatment of the disease at the beginning is usually useful, but when the symptoms persist, the treatment fails or its adverse reactions are not tolerated, it is necessary to consider alternatives such as deep brain stimulation.

Methodology: This is a narrative review with emphasis on the clinical aspects of deep brain stimulation therapy in patients with Parkinson's disease.

Discussion: Deep brain stimulation is a surgical technique in which electrodes are implanted in specific brain regions, usually the subthalamic nucleus, globus pallidus interna or ventral intermediate nucleus of the thalamus, and are connected to a subcutaneous pacemaker from which the activity of these areas is modulated electrically. This therapy has been shown to be cost-effective, provides considerable benefits in improving the symptoms of Parkinson's disease and has clinical evidence in patients who have been correctly selected.

\section{KEYWORDS}

Electric Stimulation Therapy; Neurosurgery; Parkinson Disease

\section{INTRODUCCIÓN}

La enfermedad de Parkinson (EP) es una condición neurodegenerativa progresiva que se caracteriza por presentar alteraciones en la función motora como bradicinesia, rigidez, temblor en reposo, alteraciones en la marcha e inestabilidad postural, además de manifestaciones no motoras que pueden preceder a las alteraciones motoras. La terapia farmacológica en la EP logra impactar en los síntomas de la enfermedad durante los primeros años, pero luego se hace más difícil conseguir esta mejoría debido a la aparición de complicaciones como las fluctuaciones motoras y las discinesias y por esta razón se ha venido considerando otras aproximaciones terapéuticas para el paciente con EP avanzada, entre las que se incluye la estimulación cerebral profunda (ECP).

Luego de demostrar sus efectos sobre la mejoría del temblor, la ECP se postuló como una gran alternativa para el manejo de pacientes con EP de difícil control, logrando mejorías iguales o mayores en las complicaciones motoras cuando se la comparaba con la terapia farmacológica sola o con otras intervenciones quirúrogicas (1). Actualmente, la ECP es un tratamiento ampliamente extendido para el manejo de la EP avanzada, y es una opción terapéutica en otros trastornos del movimiento y algunas patologías psiquiátricas (2).

Esta es una revisión narrativa que describe la importancia de la EP y se hace énfasis en los aspectos clínicos de la ECP, se incluye el mecanismo por el que este último mejora los síntomas de la EP, así como los efectos adversos, costos, selección de pacientes y la evidencia clínica que soporta el uso de esta terapia en quienes padecen esta condición.

\section{Enfermedad de Parkinson}

La EP es la segunda causa de enfermedad neurodegenerativa crónica progresiva (3) y es el tipo de parkinsonismo más prevalente (4). Aproximadamente 10 millones de personas en el mundo se ven afectadas por esta enfermedad, se presenta en casi el $1 \%$ de la población mayor de 65 años y un 4 a $5 \%$ de los mayores de 85 años (4-7). La prevalencia global de EP es aproximadamente 213 por cada 100.000 habitantes (8) y aumenta considerablemente después de los 70 años, llegando a ser mayor a los 1000 casos por cada 100.000 habitantes (9). En Colombia la EP afecta a 4,7 personas por cada mil habitantes (10).

Esta enfermedad se caracteriza por presentar signos $y$ síntomas tanto motores como no motores. Los síntomas motores incluyen temblor, bradicinesia-acinesia, rigidez e inestabilidad postural $(11,12)$ y el subtipo clínico más frecuente es el temblor con inestabilidad de la marcha (13). Estos síntomas suelen ser de inicio y evolución asimétrica, inician en miembros superiores 
y se hacen bilaterales en un promedio de tres años $(12,13)$. En las etapas avanzadas, se presentan manifestaciones refractarias al tratamiento como discinesias, fluctuaciones motoras, trastornos de la postura, marcha, disfagia, y disartria (14). Los síntomas no motores pueden preceder a la clínica motora e incluyen trastornos del sueño, síntomas autonómicos, gastrointestinales, cognitivos, dolor y otros $(5,14)$. A medida que avanza la enfermedad se hacen evidentes síntomas psiquiátricos como ludopatía, hipersexualidad, compras compulsivas, agresión, celos y fobias, principalmente en aquellos que abusan de fármacos dopaminérgicos $(14,15)$.

Se ha estimado que la vida media de supervivencia de los pacientes con EP está entre 11 y 15,8 años (1618). En los últimos años ha habido un aumento progresivo de mortalidad, lo que ha hecho que se ubique entre 0,9 a 3,8 por cada 100.000 habitantes $(16,19-21)$ y está asociada a comorbilidades como la neumonía, enfermedades cardiovasculares y cáncer (17, 22, 23). La calidad de vida de los afectados por EP y de su familia se deteriora conforme esta progresa, puesto que por un lado aumenta la carga de la enfermedad y por el otro, se hace necesaria la presencia de un cuidador $(24,25)$. Para mejorar este panorama se han propuesto diversos tratamientos farmacológicos y quirúrogicos (26-28), además de la ECP, que al respecto ha demostrado un impacto positivo (29-31).

\section{Principios de la ECP}

Este procedimiento ha sido ampliamente usado en pacientes con EP en quienes los síntomas no cesan, la terapia farmacológica ha fallado o no se toleran sus efectos adversos $(32,33)$. Esta es una técnica de neuromodulación en la que se implanta uno o más electrodos de cuatro contactos en regiones cerebrales específicas, estos se conectan a un marcapaso subcutáneo implantado en el pecho desde donde se modula eléctricamente los patrones de despolarización, repolarización y conducción del potencial de acción de las neuronas (34). Este tipo de intervenciones se viene explorando desde hace ya algunas décadas $(34,35)$, pero fue solo en los noventa que se estableció como un abordaje quirúrgico en humanos. Posteriormente se definió con mayor exactitud los sitios, tamaño e intensidad $(36,37)$, mejorando así las actividades motoras, las capacidades cognitivas y emocionales, al disminuir los efectos adversos secundarios a la estimulación eléctrica de zonas adyacentes $(38,39)$. Actualmente, la FDA (Food and Drug Administration) ha aprobado la modulación por ECP en el núcleo subtalámico (NST), globo pálido interno (GPI) y núcleo ventral intermedio del tálamo (NVIT) (40).

En la EP existe una pérdida progresiva de neuronas dopaminéroicas de la sustancia nigra (SN) pars compacta (41). Estas forman parte de la vía nigroestriatal que se proyecta hacia los ganglios basales en donde regulan la actividad de neuronas estriatales, cuyas eferencias modulan la actividad del tálamo de forma directa o indirecta (42). Debido a la degeneración de estas neuronas dopaminéroicas, existe un predominio de la vía indirecta en donde las eferencias nioroestriatales inhiben las neuronas GABAéroicas del globo pálido externo, Ilevando a una desinhibición del NST. Éste a su vez envía proyecciones glutamatéroicas hacia la SN pars reticulada y GPI generando la liberación de GABA sobre los núcleos talámicos que se proyectan hacia la corteza cerebral (43), lo que en últimas resulta en una disminución de la actividad motora y la consiguiente bradicinesia característica de la EP La vía directa también juega un papel importante en la enfermedad, la disminución de la liberación de dopamina por las neuronas dopaminérgicas de la SN da lugar a la inhibición de las neuronas estriatales, que a su vez inhiben el GPI y la SN pars reticulata, por lo que el tálamo queda libre de inhibición y puede enviar impulsos excitatorios a la corteza motora que se traducen en aumento de la actividad motora en forma de temblor, (Figura 1) (43-45).

La ECP en eI NST interrumpe la sincronización neuronal de la vía indirecta que conecta este núcleo con la corteza motora (46), esto aumenta el procesamiento de información sensitiva e iniciación del movimiento al incrementar la activación de la corteza premotora y por la modulación del circuito cerebelo-tálamocorteza que está conectado funcionalmente al circuito corteza-ganglios basales, (Tabla 1) (47). Asimismo, mejora las capacidades cognitivas, la memoria visual, el reconocimiento de rostros al activar el giro frontal y temporal inferior (48). Pese a esto, se han reportado aumentos en la apatía y cambios en el estado de ánimo de los pacientes intervenidos (49). 


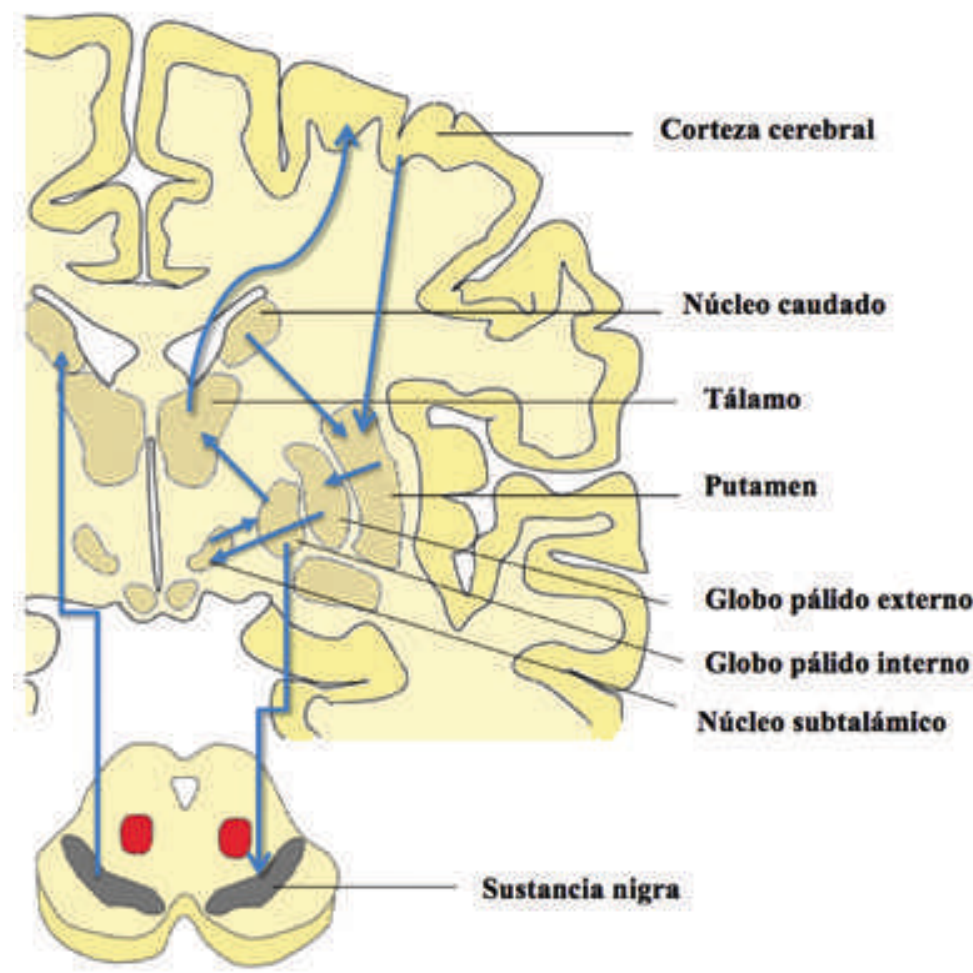

Figura 1. Principales vías de transmisión involucradas en la enfermedad de Parkinson.

Se esquematizan las principales conexiones de la vía indirecta y directa que provocan los síntomas de la enfermedad

Tabla 1. Efectos generales a nivel funcional y clínico de los núcleos cerebrales involucrados en la estimulación cerebral profunda

\begin{tabular}{|c|c|c|}
\hline Núcleo & Efecto neuronal & Efecto clínico \\
\hline $\begin{array}{l}\text { Núcleo subtalámico } \\
\text { (NST) }\end{array}$ & $\begin{array}{l}\text { Interfiere comunicación entre } \\
\text { NST y corteza motora primaria. }\end{array}$ & $\begin{array}{l}\text { - Mejora: procesamiento señales sensitivas. } \\
\text { - Mejora: inicio del movimiento. } \\
\text { - Mejora: capacidades verbales. } \\
\text { - Mejora: memoria visual, reconocimiento rostros. }\end{array}$ \\
\hline Globo pálido interno & $\begin{array}{l}\text { Modulación: vía directa e indi- } \\
\text { recta de ganglios basales. }\end{array}$ & $\begin{array}{l}\text { - Inhibición de la distonia. } \\
\text { - Mejoría en la deglución. } \\
\text { - Mejoría en el balance y la marcha. }\end{array}$ \\
\hline Núcleo ventral intermedio del tálamo & $\begin{array}{l}\text { Disminuye actividad área pre- } \\
\text { motora, asociación, sensitivo- } \\
\text { motoras primarias. }\end{array}$ & $\begin{array}{l}\text { Disminuye el temblor esencial. } \\
\text { - Disminuye el temblor refractario a la terapia. }\end{array}$ \\
\hline
\end{tabular}


En el GPI converge la vía inhibitoria directa e indirecta de los ganglios basales, su estimulación con ECP genera una inhibición de la distonía generada por la enfermedad y mejora el balance, la marcha y la deglución (50). La neuromodulación que se realiza en el NVIT disminuye la actividad en el área premotora, de asociación, sensitivo-motoras primarias y en el cerebelo contralateral modulando la amplitud, frecuencia $y$ regularidad del temblor esencial $y$ otros tipos de temblores refractarios a la terapia (51). El efecto es aún mayor cuando se estimula el mismo sitio bilateralmente, en cuyo caso puede durar hasta 7 años (52). Otros sitios explorados son el núcleo pedúnculo pontino, ventrolateral, ventral anterior $y$ centromedial del tálamo $(40,53)$.

El efecto a nivel celular lo condiciona la composición y propiedades de las membranas que están en contacto con las frecuencias o por señales eléctricas que dicta el electrodo. En etapas agudas la estimulación produce una respuesta glial e inflamatoria contra el electrodo, las neuronas pueden generar potenciales excitatorios o inhibitorios en sentido ortodrómico o antidrómico, lo que permite la normalización de la frecuencia del potencial de propagación del estímulo en las vías ascendentes y descendentes de los núcleos estimulados $(54,55)$. Además, se cambian las frecuencias y el flujo sanguíneo cerebral en las regiones bajo la influencia del estímulo eléctrico $(33,55)$. La microglía y los astrocitos promueven la angiogénesis y la reorganización sináptica que se da con el tiempo, haciendo que el efecto crónico de la estimulación genere cambios en la plasticidad sináptica, generación de neuritas, aumento en la densidad sináptica y cambios en la actividad metabólica de las células (56).

Después de que se activa la ECP, el curso y los patrones de mejoría en los síntomas varían considerablemente entre los síntomas que pueden ser tratados mediante la neuroestimulación. El temblor y la rigidez suelen responder rápidamente en minutos, mientras que la bradicinesia puede tardar horas, la distonía y el dolor mejoran en meses, los cambios comportamentales y neurocognitivos aparecen también en el transcurso de meses (57).

\section{Costos}

Se estima que los costos médicos directos por consulta externa, como horas de personal de salud, insumos, medicamentos, entre otros, avalúan un promedio de 485,74 dólares americanos al mes por sujeto (58), que podría alcanzar hasta 5.993 euros al año (59). Los costos cambian de acuerdo al tipo de tratamiento realizado, se habla de un costo promedio de vida de 126.200 euros en pacientes con tratamiento médico estándar hasta 133.200 euros para pacientes con ECP (60), otros reportan un costo promedio a 5 años de 186.244 dólares (61), incluso se habla de una reducción de hasta el $40 \%$ promedio de los costos por medicamentos después de la implantación $(31,61)$.

En relación al dispositivo, el mayor costo se ve reflejado en el momento de la implantación con cerca de 4.184 dólares hasta alcanzar 29.178 dólares en el primer año y a partir de allí un aumento promedio de 1.490 dólares por año. El mayor costo en el primer año es debido al valor de dispositivo, la intervención quirúrgica y los controles (62), pero a partir de ahí muestra ser un tratamiento costo-efectivo y por lo tanto la ECP es una alternativa en el manejo de la EP en estados avanzados $(60,62-64)$.

\section{Efectos adversos}

Las infecciones tienen una prevalencia de 0 a $15 \%$ $(65,66)$, siendo el Staphylococcus aureus el principal agente etiológico $(67,68)$. La hemorragia intracraneal, en especial la hemorragia intraventricular o intracerebral, localizadas a lo largo del trayecto de implantación del dispositivo, pueden ocurrir hasta en el $10 \%$ de los casos (66) pero generalmente son asintomáticas y auto limitadas (65), aunque se han reportado de forma aislada hemiparesias resueltas con terapia física (68).

Los efectos adversos neurológicos son rigidez postoperatoria, convulsiones parciales, confusión y eventos cerebrovasculares $(66,67)$. Las discinesias después de encender el dispositivo son un hallazgo normal y de buen pronóstico porque se asocian a un efecto sinérgico entre la medicación y la intervención quirúrgica (65). Alounos efectos neuropsicológicos han sido documentados, entre ellos la disminución selectiva de las funciones cognitivas frontales, en la fluidez verbal y en el desarrollo del test de Stroop (69-71). Se ha reportado una disminución de la función cognitiva global durante los primeros seis meses después de la implantación, en comparación con los siguientes 6 a 
36 meses, debido posiblemente, a la reducción excesiva de levodopa en los primeros meses posquirúrogicos (72). También se han documentado trastornos mentales como comportamientos maníacos, hipomaniacos o depresivos que se manifiestan en los primeros seis meses posquirúrgicos pero muestran una mejoría progresiva $(65,69,73,74)$.

Otros efectos posquirúrquicos adversos reportados son la trombosis venosa profunda, aumento del índice de masa corporal, erosión de la piel en la zona del generador de impulsos interno (1-2,5\%), migración del dispositivo (0-19\%), rotura o fractura en la extensión del cable del dispositivo (0-15\%), adherencias alrededor de éste e incluso la muerte $(65,66,68,74-76)$.

En su mayoría, tales reacciones adversas no son dependientes del centro donde se realice el procedimiento quirúrgico, ya sea con alto o bajo flujo de pacientes con EP (77). El habla, la expresión facial, la bradicinesia, el temblor y los síntomas axiales hacen parte de los signos y síntomas propios de la EP, que mejoran con el encendido del dispositivo, pero que reaparecen una vez este se apaga $(65,78,79)$.

\section{Selección de pacientes}

Se debe establecer una relación riesgo-beneficio para determinar si un paciente con EP se beneficiará con la terapia quirúroica (80). Centros especializados han establecido los criterios para seleccionar a quienes se podrían beneficiar de la ECP, estos consideran el estado del paciente, las complicaciones asociadas al tratamiento, entre otras, (Tabla 2) $(33,81,82)$. Sin embargo, su aplicación reduce sustancialmente el número de inclusión $(1,6 \%)$, porcentaje que podría aumentarse con otros más flexibles (83) debido a que aún se encuentra en debate alounos aspectos relacionados con el tema (82).

\section{Tabla 2. Criterios de selección en pacientes con enfermedad de Parkinson}

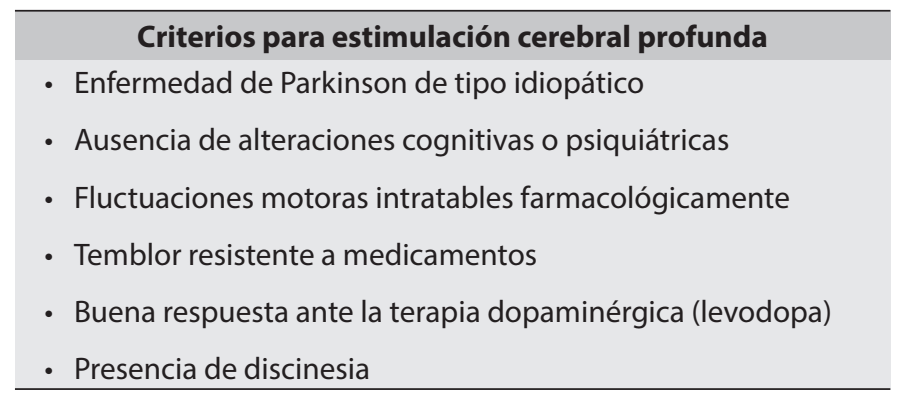

\section{Evidencia clínica}

A lo largo de los años la ECP ha demostrado beneficios en el tratamiento de los pacientes con EP. La seguridad $y$ eficacia de esta terapia son temas claves y controversiales en las investigaciones, ya que proporcionan argumentos para el médico tratante a la hora de tomar una decisión al respecto. Por eso este apartado busca orientar al lector hacia algunos de los hallazgos clínicos relevantes sobre el tema.

Para comparar la ECP deI NST más terapia farmacológica (ECP-F) versus el tratamiento farmacológico solo, se realizó un ensayo aleatorizado donde se incluyeron 156 pacientes con EP avanzada y síntomas motores severos. Se evaluaron los cambios en la calidad de vida según el cuestionario PDQ-39 y la severidad de los síntomas sin medicación, de acuerdo con la escala UPDRS-III. La ECP-F en comparación con la terapia farmacológica sola, causó mejorías desde el inicio hasta seis meses en el PDQ-39 y la UPDRS-III, con una mejoría media de 9,5 y 19,6 puntos, respectivamente. La neuroestimulación resultó en un incremento de $24 \%$ al $38 \%$ en las subescalas para la movilidad $(p<0,001)$, actividades de la vida diaria $(p<0,001)$, el bienestar emocional $(p<0,001)$, el estigma $(p<0,001)$ y malestar corporal $(p=0,009)$. Los eventos adversos graves fueron más frecuentes con la neuroestimulación que con la medicación sola $(12,8$ 
$\%$ frente al 3,8 \%, p=0,04) e incluía una hemorragia intracerebral fatal (84).

Un ensayo clínico aleatorizado reunió a un total de 255 pacientes con EP avanzada y comparó el efecto de la ECP contra la terapia farmacológica. A 60 se les realizó ECP bilateral deI NST, a 61 ECP del GPI y 134 recibieron tratamiento farmacológico. En relación con el primer grupo, estos últimos presentaron más tiempo libre de discinesias (media de diferencia entre los grupos 4,5 horas/día; IC 95 \% 3,7-5,4; p<0,001). Además, comparado con el grupo que recibió el tratamiento farmacológico a los 6 meses (media de diferencia entre los grupos 10,6; IC $95 \%$ 8,1-13,2; $\mathrm{p}<0,001$ ), se encontró que en el grupo con ECP, mientras no tomaba medicación, turo una mejoría en 12,3 puntos en la función motora y se reveló en los exámenes neurocognitivos pequeñas disminuciones en el desempeño en estas pruebas, mientras que el grupo de la terapia farmacológica tuvo leves mejorías (31).

En un ensayo clínico aleatorizado pero con una muestra limitada de 30 pacientes se comparó el tratamiento farmacológico solo versus ECP-F y se encontró que en el grupo sometido a ECP-F ocurrieron dos efectos adversos (evento cerebrovascular y hardware infectado) y una disminución en cuanto a la fluidez verbal $(\mathrm{p}=0,047)$, aunque al compararlo con el grupo de solo terapia farmacológica no hay diferencia sionnificativa si se excluyen estos efectos adversos (a los 12 meses de seguimiento: $\mathrm{p}=0,066$ y a los 24 meses: $\mathrm{p}=$ $0,096)(85)$. Otro estudio con el mismo número de pacientes al anterior y con EP temprana, encontró que al comparar la seguridad de la terapia farmacológica con la ECP-F, este segundo grupo requirió menos medicación antiparkinsoniana durante todo el estudio y que a 24 meses no hubo empeoramiento de la función motora (86).

En cuanto a los síntomas no motores, se ha encontrado que en un principio la ECP mejora la ansiedad, pero en el seguimiento a tres meses no existieron cambios significativos e incluso continuó empeorando $(p<0,05)$, por lo que se concluye que ni la terapia farmacológica ni la ECP pueden evitar la progresión de la EP $(87,88)$.

Las distintas zonas blanco para la neuroestimulación han sido comparadas, es el caso de la estimulación del GPI vs NST en los efectos sobre la postura corporal, que utilizó como grupo control individuos sanos $y$ pacientes que eran candidatos para la cirugía pero decidieron no someterse al procedimiento. Se descubrió que la estimulación tanto del GPI como del NST mejoran la estabilidad postural en el postoperatorio sin medicación ( $p=0.002)$, sin embaroo, al comparar aquellos pacientes con adecuado tratamiento farmacológico frente a aquellos con estimulación deI NST y medicación, estos últimos presentaron una peor estabilidad postural a los 6 meses $(p=0.012)$, asociado, probablemente, al mayor número de caídas en pacientes con estimulación del NST (89).

Por otro lado, el ensayo clínico NSTAPS demostró que tanto la estimulación del NST y del GBI mejoran los síntomas motores, pero al compararlos, fue mayor la mejoría con estimulación del NST $(p=0.04)$ y se requirió menor dosis de levodopa en este grupo $(\mathrm{p}<0.001)$. Asimismo, se halló que para ambos blancos la mejoría persiste en el tiempo al seguirlos por 3 años $(90,91)$.

Al momento no se han encontrado diferencias clínicamente sionificativas en los resultados neuropsicológicos de la ECP-NST y ECP-GPI (71). Un estudio demostró, en cuanto a la seguridad de la ECP, que los eventos adversos graves se produjeron en el $51 \%$ de ECP-GPI y en el $56 \%$ de las personas sometidas a ECPNST, sin diferencias sionificativas entre los grupos a los 24 meses ( $p>0,05$ en todos los reportados) (50).

\section{CONCLUSIONES}

La ECP es una terapia eléctrica que busca estimular zonas específicas en el cerebro con el fin de disminuir los síntomas de la EP. Los pacientes que pueden beneficiarse son aquellos con complicaciones motoras o discinesias debidas al tratamiento farmacológico o en aquellos que no tienen mejoría en sus síntomas, especialmente en el temblor. En ellos, la ECP ha mostrado ser una alternativa costo-efectiva, con una frecuencia de eventos adversos baja y con mejorías en los síntomas motores y no motores.

\section{CONFLICTOS DE INTERESES}

Ninguno por declarar. 


\section{REFERENCIAS BIBLIOGRÁFICAS}

1. Miocinovic S, Somayajula S, Chitnis S, Vitek JL. History, applications, and mechanisms of deep brain stimulation. JAMA Neurol. 2013 Feb;70(2):163-71. DOI 10.1001/2013.jamaneurol.45.

2. Sharma M, Naik $\nabla$, Deogaonkar M. Emerging applications of deep brain stimulation. J Neurosurg Sci. 2016 Jun;60(2):242-55.

3. Schuepbach WM, Rau J, Knudsen K, Volkmann J, Krack P, Timmermann L, et al. Neurostimulation for Parkinson's disease with early motor complications. N Engl J Med. 2013 Feb;368(7):610-22. DOI 10.1056/ NEJMoa 1205158.

4. Fereshtehnejad SM, Shafieesabet M, Rahmani A, Delbari A, Lökk J. Medium-to-high prevalence of screening-detected parkinsonism in the urban area of Tehran, Iran: data from a community-based door-to-door study. Neuropsychiatr Dis Treat. 2015 Feb;11:321-32. DOI 10.2147/NDT.S77391.

5. Moreno CB, Hernández-Beltrán N, Munevar D, Gutiérrez-Álvarez AM. Central neuropathic pain in Parkinson's disease. Neurologia (Barcelona, Spain). 2012 Oct;27(8):500-3. DOI 10.1016/j.nrleng. 2011.08.002.

6. de Lau LM, Breteler MM. Epidemiology of Parkinson's disease. Lancet Neurol. 2006 Jun;5(6):525-35.

7. de Rijk MC, Tzourio C, Breteler MM, Dartigues JF, Amaducci L, Lopez-Pousa S, et al. Prevalence of parkinsonism and Parkinson's disease in Europe: the EUROPARKINSON Collaborative Study. European Community Concerted Action on the Epidemiology of Parkinson's disease. J Neurol Neurosuro Psychiatry. 1997 Jan;62(1):10-5.

8. El-Tallawy HN, Farghaly WM, Shehata GA, Rageh TA, Hakeem NM, Hamed MA, et al. Prevalence of Parkinson's disease and other types of Parkinsonism in Al Kharga district, Egypt. Neuropsychiatr Dis Treat. 2013:9:1821-6. DOI 10.2147/NDT.S48318.

9. Pringsheim T, Jette N, Frolkis A, Steeves TD. The prevalence of Parkinson's disease: a systematic review and meta-analysis. Mov Disord. 2014 Nov;29(13):1583-90. DOI 10.1002/mds.25945.

10. Pradilla G, Boris E, Vesga A, León-Sarmiento FE; Grupo GENECO. Estudio neuroepidemiológico nacional (EPINEURO) colombiano. Rev Panam Salud Pública. 2003;14(2):104-11.
11. Cervantes-Arriaga A, Rodríguez-Violante M, LópezRuiz M, Estrada-Bellmann I, Zuñiga-Ramírez C, Otero-Cerdeira E, et al. Caracterización de la enfermedad de Parkinson en México: estudio ReMePARK. Gac Méd Mex. 2013;149:497-501.

12. Torres-Ramírez L, Villafuerte-Espinoza M, CosentinoEsquerre C, Flores-Mendoza M, Vélez-Rojas M, Suárez-Reyes R, et al. Características clínicas de la enfermedad de Parkinson en una cohorte de pacientes peruanos. Rev Per Neurol. 2012;13(1):4-9.

13. Lo RY, Tanner CM, Albers KB, Leimpeter AD, Fross $\mathrm{RD}$, Bernstein AL, et al. Clinical features in early Parkinson disease and survival. Arch Neurol. 2009 Nov;66(11):1353-8. DOI 10.1001/archneurol.2009.221.

14. Kulisevsky J, Luquin M, Arbelo J, Burouera J, Carrillo F, Castro A, et al. Enfermedad de Parkinson avanzada. Características clínicas y tratamiento (parte I). Neurologia (Barcelona, Spain). 2013;28(8):503-21. DOI 10.1016/j.nrl.2013.05.001.

15. Wu K, Politis M, Piccini P. Parkinson disease and impulse control disorders: a review of clinical features, pathophysiology and management. Postgrad Med J. 2009 Nov;85(1009):590-6. DOI 10.1136/ pogmj.2008.075820.

16. Das SK, Misra AK, Ray BK, Hazra A, Ghosal MK, Chaudhuri A, et al. Epidemiology of Parkinson disease in the city of Kolkata, India: a community-based study. Neurology. 2010 Oct;75(15):1362-9. DOI 10.1212/ WNL.0bo13e3181f735a7.

17. Duarte J, García OImos LM, Mendoza A, Clavería LE. The natural history of Parkinson's disease in the province of Segovia: mortality in a longitudinal study (20-year follow-up). Acta Neurol Scand. 2013 May;127(5):295-300. DOI 10.1111/ane.12003.

18. Forsaa EB, Larsen JP, Wentzel-Larsen T, Alves G. What predicts mortality in Parkinson disease?: a prospective population-based long-term study. Neurology. 2010 Oct;75(14):1270-6. DOI 10.1212/ WNL.0b013e3181f61311.

19. Chaná P, Jiménez M, Díaz $\nabla$, Juri C. Mortalidad por enfermedad de Parkinson en Chile. Rev Méd Chi1e. 2013 Mar;141(3):327-31. DOI 10.4067/S003498872013000300007 .

20. Pérez Pastene C, Varogas Rona C, Silva Opazo J, Cortés Arancibia S. Comportamiento de la mortalidad por la Enfermedad de Parkinson en Chile en el periodo 1990-2009. Acta Neurol Colomb. 2014;30(2):97-102. 
21. Macleod AD, Taylor KS, Counsell CE. Mortality in Parkinson's disease: a systematic review and metaanalysis. Mov Disord. 2014 Nov;29(13):1615-22. DOI 10.1002/mds. 25898 .

22. Fernandes GC, Socal MP, Schuh AF, Rieder CR. Clinical and Epidemiological Factors Associated with Mortality in Parkinson's Disease in a Brazilian Cohort. Parkinsons Dis. 2015;2015:959304. DOI 10.1155/2015/959304.

23. Pennington S, Snell K, Lee M, Walker R. The cause of death in idiopathic Parkinson's disease. Parkinsonism Relat Disord. 2010 Aug;16(7):434-7. DOI 10.1016/j. parkreldis.2010.04.010.

24. Rodríguez-Violante M, Camacho-Ordoñez A, Cervantes-Arriaga A, González-Latapí P, Velázquez-Osuna S. Factores asociados a la calidad de vida de sujetos con enfermedad de Parkinson ya la carga en el cuidador. Neurologia (Barcelona, Spain). 2015;30(5):257-63. DOI 10.1016/j.nrI.2014.01.008.

25. Leiknes I, Tysnes OB, Aarsland D, Larsen JP. Caregiver distress associated with neuropsychiatric problems in patients with early Parkinson's disease: the Norwegian ParkWest study. Acta Neurol Scand. 2010 Dec;122(6):418-24. DOI 10.1111/j.16000404.2010.01332.x.

26. McNeely ME, Duncan RP, Earhart GM. Impacts of dance on non-motor symptoms, participation, and quality of life in Parkinson disease and healthy older adults. Maturitas. 2015 Dec;82(4):336-41. DOi 10.1016/j.maturitas.2015.08.002.

27. Yousefi B, Tadibi $\nabla$, Khoei AF, Montazeri A. Exercise therapy, quality of life, and activities of daily living in patients with Parkinson disease: a small scale quasi-randomised trial. Trials. 2009 Aug;10:67. DOI 10.1186/1745-6215-10-67.

28. Cholewa J, Boczarska-Jedynak M, Opala G. Influence of physiotherapy on severity of motor symptoms and quality of life in patients with Parkinson disease. Neurol Neurochir Pol. 2013 May-Jun;47(3):256-62.

29. Pla Casamitjana C, García S, Zárate Méndez A, Hernández Salazar M, Sauri Suárez S, Meza Dávalos E, et al. Calidad de vida en pacientes con enfermedad de Parkinson y estimulación cerebral profunda. Med Int Mex. 2007;23:7-14.

30. Perestelo-Pérez L, Rivero-Santana A, Pérez-Ramos J, Serrano-Pérez P, Panetta J, Hilarion P. Deep brain stimulation in Parkinson's disease: meta-analysis of randomized controlled trials. J Neurol. 2014 Nov;261(11):2051-60. DOI 10.1007/s00415-014-7254-6.

31. Weaver FM, Follett K, Stern M, Hur K, Harris C, Marks WJ Jr, et al. Bilateral deep brain stimulation $\nabla \mathrm{s}$ best medical therapy for patients with advanced Parkinson disease: a randomized controlled trial. JAMA. 2009 Jan;301(1):63-73. DOI 10.1001/jama.2008.929.

32. Fox SH, Katzenschlager R, Lim SY, Ravina B, Seppi K, Coelho M, et al. The Movement Disorder Society Evidence-Based Medicine Review Update: Treatments for the motor symptoms of Parkinson's disease. Mov Disord. 2011 Oct;26 SuppI 3:S2-41. DOI 10.1002/ mds.23829.

33. Okun MS. Deep-Brain Stimulation for Parkinson's Disease. N Engl J Med. 2012;367(16):1529-38. DOI 10.1056/NEJMct1208070.

34. Hickey P, Stacy M. Deep Brain Stimulation: A Paradigm Shifting Approach to Treat Parkinson's Disease. Front Neurosci. 2016 Apr;10:173. DOI 10.3389/ fnins.2016.00173.

35. Gionfriddo MR, Greenberg AJ, Wahegaonkar AL, Lee KH. Pathways of translation: deep brain stimulation. Clin TransI Sci. 2013 Dec;6(6):497-501. DOI 10.1111/cts.12055. Erratum in: Clin TransI Sci. 2014 Jun; $7(3): 284$

36. Pote I, Torkamani M, Kefalopoulou ZM, Zrinzo L, Limousin-Dowsey P, Foltynie T, et al. Subthalamic nucleus deep brain stimulation induces impulsive action when patients with Parkinson's disease act under speed pressure. Exp Brain Res. 2016 Jul;234(7):18371848. DOI 10.1007/s00221-016-4577-9.

37. Xie CL, Shao B, Chen J, Zhou Y, Lin SY, Wang WW. Effects of neurostimulation for advanced Parkinson's disease patients on motor symptoms: A multipletreatments meta-analysas of randomized controIled trials. Sci Rep. 2016 May;6:25285. DOI 10.1038/ srep25285.

38. Okun MS, Gallo BV, Mandybur G, Jagid J, Foote KD, Revilla FJ, et al. Subthalamic deep brain stimulation with a constant-current device in Parkinson's disease: an open-label randomised controlled trial. Lancet Neurol. 2012 Feb;11(2):140-9. DOI 10.1016/S14744422(11)70308-8. Erratum in: Lancet Neurol. 2012 Mar;11(3):208.

39. Williams A, Gill S, Varma T, Jenkinson C, Quinn N, Mitchell R, et al. Deep brain stimulation plus best 
medical therapy versus best medical therapy alone for advanced Parkinson's disease (PD SURG trial): a randomised, open-label trial. Lancet Neurol. 2010 Jun;9(6):581-91. DOI 10.1016/S1474-4422(10)70093-4.

40. DeLong MR, Wichmann T. Basal Ganglia Circuits as Targets for Neuromodulation in Parkinson Disease. JAMA Neurol. 2015 Nov;72(11):1354-60. DOI 10.1001/ jamaneurol.2015.2397.

41. Damier P, Hirsch EC, Agid Y, Graybiel AM. The substantia nigra of the human brain. II. Patterns of loss of dopamine-containing neurons in Parkinson's disease. Brain. 1999 Aug; 122 ( Pt 8):1437-48.

42. Sarkar S, Raymick J, Imam S. Neuroprotective and Therapeutic Strategies against Parkinson's Disease: Recent Perspectives. Int J Mol Sci. 2016 Jun;17(6). pii: E904. DOI 10.3390/ijms17060904.

43. Calabresi P, Picconi B, Tozzi A, Ghiglieri V, Di Filippo M. Direct and indirect pathways of basal ganglia: a critical reappraisal. Nat Neurosci. 2014 Aug; 17(8):1022-30. DOI 10.1038/nn.3743.

44. DeLong MR, Wichmann T. Circuits and circuit disorders of the basal ganglia. Arch Neurol. 2007 Jan;64(1):20-4.

45. Gerfen CR, Surmeier DJ. Modulation of striatal projection systems by dopamine. Annu Rev Neurosci. 2011;34:441-66. DOI 10.1146/annurev-neuro-061010-113641.

46. Alhourani A, McDowell MM, Randazzo MJ, Wozny TA, Kondylis ED, Lipski WJ, et aI. Network effects of deep brain stimulation. J Neurophysiol. 2015 Oct;114(4):2105-17. DOI 10.1152/jn.00275.2015.

47. Kahan J, Urner M, Moran R, Flandin G, Marreiros A, Mancini L, et al. Resting state functional MRI in Parkinson's disease: the impact of deep brain stimulation on 'effective' connectivity. Brain. 2014 Apr; $137(\mathrm{Pt}$ 4):1130-44. DOI 10.1093/brain/awu027.

48. Kalbe E, Voges J, Weber T, Haarer M, BaudrexeI S, Klein JC, et al. Frontal FDG-PET activity correlates with cognitive outcome after STN-DBS in Parkinson disease. Neurology. 2009 Jan;72(1):42-9. DOI 10.1212/01. wnI.0000338536.31388.fo.

49. Le Jeune F, Drapier D, Bourguignon A, Péron J, Mesbah H, Drapier S, et al. Subthalamic nucleus stimulation in Parkinson disease induces apathy: a PET study. Neurology. 2009 Nor;73(21):1746-51. DOI 10.1212/ WNL.0b013e3181c34b34.
50. Follett KA, Weaver FM, Stern M, Hur K, Harris CL, Luo p, et el. Pallidal versus subthalamic deep-brain stimulation for Parkinson's disease. N Engl J Med. 2010 Jun;362(22):2077-91. DOI 10.1056/NEJMoa0907083.

51. Mure H, Hirano S, Tang CC, Isaias IU, Antonini A, Ma Y, et al. Parkinson's disease tremor-related metabolic network: characterization, progression, and treatment effects. Neuroimage. 2011 Jan;54(2):124453. DOI 10.1016/j.neuroimage.2010.09.028.

52. Pandey S, Sarma N. Deep brain stimulation: current status. Neurol India. 2015 Jan-Feb;63(1):9-18. DOI 10.4103/0028-3886.152623.

53. Anderson D, Beecher G, Ba F. Deep Brain Stimulation in Parkinson's Disease: New and Emerging Targets for Refractory Motor and Nonmotor Symptoms. Parkinsons Dis. 2017;2017:5124328. DOI 10.1155/2017/5124328.

54. PerImutter JS, Mink JW. Deep brain stimulation. Annu Rev Neurosci. 2006;29:229-57.

55. Agnesi F, Johnson MD, Vitek JL. Deep brain stimulation: how does it work? Handb Clin Neurol. 2013;116:3954. DOI 10.1016/B978-0-444-53497-2.00004-8.

56. van HarteveIt TJ, Cabral J, Deco G, Møller A, Green AL, Aziz TZ, et al. Neural plasticity in human brain connectivity: the effects of long term deep brain stimulation of the subthalamic nucleus in Parkinson's disease. PLoS One. 2014 Jan;9(1):e86496. DOI 10.1371/ journal.pone.0086496.

57. Ashkan K, Rogers P, Bergman H, Ughratdar I. Insights into the mechanisms of deep brain stimulation. Nat Rev Neurol. 2017 Sep;13(9):548-554. DOI 10.1038/nrneurol.2017.105.

58. Yoritaka A, Fukae J, Hatano T, Oda E, Hattori N. The Direct Cost of Parkinson Disease at Juntendo Medical University Hospital, Japan. Intern Med. 2016;55(2):1139. DOI 10.2169/internalmedicine.55.4484.

59. Findley LJ. The economic impact of Parkinson's disease. Parkinsonism Relat Disord. 2007 Sep;13 Suppl:S8-S12.

60. Dams J, Siebert U, Bornschein B, Volkmann J, Deuschl G, Oertel WH, et al. Cost-effectiveness of deep brain stimulation in patients with Parkinson's disease. Mov Disord. 2013 Jun;28(6):763-71. DOI 10.1002/ mds. 25407.

61. Valldeoriola F, Morsi O, Tolosa E, Rumià J, Martí MJ, Martínez-Martín P. Prospective comparative study 
on cost-effectiveness of subthalamic stimulation and best medical treatment in advanced Parkinson's disease. Mov Disord. 2007 Nov;22(15):2183-91.

62. Zhu XL, Chan DT, Lau CK, Poon WS, Mok VC, Chan AY, et al. Cost-effectiveness of subthalmic nucleus deep brain stimulation for the treatment of advanced Parkinson disease in Hong Kong: a prospective study. World Neurosurg. 2014 Dec;82(6):987-93. DOI 10.1016/j.wneu.2014.08.051.

63. Kawamoto Y, Mouri M, Taira T, Iseki H, Masamune K. Cost-Effectiveness Analysis of Deep Brain Stimulation in Patients with Parkinson's Disease in Japan. World Neurosurg. 2016 May;89:628-635.e1. DOI 10.1016/j. wneu.2015.11.062.

64. Eggington S, Valldeoriola F, Chaudhuri KR, Ashkan K, Annoni E, Deuschl G. The cost-effectiveness of deep brain stimulation in combination with best medical therapy, versus best medical therapy alone, in advanced Parkinson's disease. J Neurol. 2014 Jan;261(1):10616. DOI 10.1007/s00415-013-7148-z.

65. Mandat T, Tykocki T, Koziara H, Koziorowski D, Brodacki B, Rola R, et al. Subthalamic deep brain stimulation for the treatment of Parkinson disease. Neurol Neurochir Pol. 2011 Jan-Feb;45(1):32-6.

66. Sharma A, Szeto K, Desilets AR. Efficacy and safety of deep brain stimulation as an adjunct to pharmacotherapy for the treatment of Parkinson disease. Ann Pharmacother. 2012 Feb;46(2):248-54. DOI 10.1345/aph.1Q508.

67. Parent B, Awan N, Berman SB, Suski V, Moore R, Crammond $\mathrm{D}$, et al. The relevance of age and disease duration for intervention with subthalamic nucleus deep brain stimulation surgery in Parkinson disease. J Neurosurg. 2011 Apr;114(4):927-31. DOI 10.3171/2010.10.JNS10756.

68. Vergani F, Landi A, Pirillo D, Cilia R, Antonini A, Sganzerla EP Surgical, medical, and hardware adverse events in a series of 141 patients undergoing subthalamic deep brain stimulation for Parkinson disease. World Neurosurg. 2010 Apr;73(4):338-44. DOi 10.1016/j.wneu.2010.01.017.

69. Witt K, Daniels C, Reiff J, Krack P, Volkmann J, Pinsker MO, et al. Neuropsychological and psychiatric changes after deep brain stimulation for Parkinson's disease: a randomised, multicenter study. Lancet Neurol. 2008 Jul;7(7):605-14. DOI 10.1016/S14744422(08)70114-5.
70. Rothlind JC, York MK, Carlson K, Luo P, Marks WJ, Jr., Weaver FM, et al. Neuropsychological changes following deep brain stimulation surgery for Parkinson's disease: comparisons of treatment at pallidal and subthalamic targets versus best medical therapy. Journal of neurology, neurosurgery, and psychiatry. 2015;86(6):622-9.

71. Odekerken VJ, Boel JA, Geurtsen GJ, Schmand BA, Dekker IP, de Haan RJ, et al. Neuropsychological outcome after deep brain stimulation for Parkinson disease. Neurology. 2015 Mar;84(13):1355-61. DOI 10.1212/WNL.0000000000001419.

72. Kim HJ, Jeon BS, Yun JY, Kim YE, Yang HJ, Paek SH. Initial cognitive dip after subthalamic deep brain stimulation in Parkinson disease. J Neurol. 2013 Aug;260(8):2130-3. DOI 10.1007/s00415-013-6959-2.

73. Krack P, Hariz MI. Parkinson disease: deep brain stimulation in Parkinson disease-what went wrong? Nat Rev Neurol. 2010 Oct;6(10):535-6. DOI 10.1038/nrneurol.2010.141.

74. Umemura A, Oka Y, Ohkita K, Yamawaki T, Yamada K. Effect of subthalamic deep brain stimulation on postural abnormality in Parkinson disease. J Neurosurg. 2010 Jun;112(6):1283-8. DOI 10.3171/2009.10. JNS09917.

75. Bannier S, Montaurier C, Derost Pp, Ulla M, Lemaire JJ, Boirie $\mathrm{Y}$, et al. Overweight after deep brain stimulation of the subthalamic nucleus in Parkinson disease: long term follow-up. J Neurol Neurosurg Psychiatry. 2009 May;80(5):484-8. DOI 10.1136/jnnp.2008.158576.

76. Zibetti M, Rosso M, Cinquepalmi A, Lanotte M, Angrisano S, Rabbia C, et al. Asymptomatic deep venous thrombosis after deep brain stimulation for Parkinson disease. Stereotact Funct Neurosurg. 2010;88(2):94-7. DOI 10.1159/000280821.

77. McGovern RA, Sheehy JP, Zacharia BE, Chan AK, Ford B, McKhann GM 2nd. Unchanged safety outcomes in deep brain stimulation surgery for Parkinson disease despite a decentralization of care. J Neurosurg. 2013 Dec;119(6):1546-55. DOI 10.3171/2013.8.JNS13475.

78. Buhmann C, Huckhagel T, Engel K, Gulberti A, Hidding $\mathrm{U}$, Poetter-Nerger $M$, et al. Adverse events in deep brain stimulation: A retrospective long-term analysis of neurological, psychiatric and other occurrences. PLoS One. 2017 JuI;12(7):e0178984. DOI 10.1371/journal.pone.0178984. 
79. Cooper SE, McIntyre CC, Fernandez HH, Vitek JL. Association of deep brain stimulation washout effects with Parkinson disease duration. JAMA Neurol. 2013 Jan;70(1):95-9. DOI 10.1001/jamaneurol.2013.581.

80. Pollak P. Deep brain stimulation for Parkinson's disease - patient selection. Handb Clin Neurol. 2013;116:97105. DOI 10.1016/B978-0-444-53497-2.00009-7.

81. Bronstein JM, Tagliati M, Alterman RL, Lozano AM, Volkmann J, Stefani A, et al. Deep brain stimulation for Parkinson disease: an expert consensus and review of key issues. Arch Neurol. 2011 Feb;68(2):165. DOI 10.1001/archneurol.2010.260.

82. Moldovan AS, Groiss SJ, Elben S, Südmeyer M, Schnitzler A, Wojtecki L. The treatment of Parkinson's disease with deep brain stimulation: current issues. Neural Regen Res. 2015 Jul;10(7):1018-22. DOI 10.4103/1673-5374.160094.

83. Morgante L, Morgante F, Moro E, Epifanio A, Girlanda p, Ragonese P, et al. How many parkinsonian patients are suitable candidates for deep brain stimulation of subthalamic nucleus? Results of a questionnaire. Parkinsonism Relat Disord. 2007 Dec;13(8):528-31.

84. Deuschl G, Schade-Brittinger C, Krack P, Volkmann J, Schäfer H, Bötzel K, et al. A randomized trial of deepbrain stimulation for Parkinson's disease. N Engl J Med. 2006 Aug 31;355(9):896-908. Erratum in: N Engl J Med. 2006 Sep;355(12):1289.

85. Tramontana MG, Molinari AL, Konrad PE, Davis TL, Wylie SA, Neimat JS, et al. Neuropsychological effects of deep brain stimulation in subjects with early stage Parkinson's disease in a randomized clinical trial. J Parkinsons Dis. 2015;5(1):151-63. DOI 10.3233/JPD140448.
86. Charles D, Konrad PE, Neimat JS, Molinari AL, Tramontana MG, Finder SG, et al. Subthalamic nucleus deep brain stimulation in early stage Parkinson's disease. Parkinsonism Relat Disord. 2014 Jul;20(7):7317. DOI 10.1016/j.parkreldis.2014.03.019.

87. Chang C, Li N, Wu Y, Geng N, Ge S, Wang J, et aI. Associations between bilateral subthalamic nucleus deep brain stimulation (STN-DBS) and anxiety in Parkinson's disease patients: a controlled study. J Neuropsychiatry Clin Neurosci. 2012 Summer;24(3):316-25. DOI 10.1176/appi.neuropsych.11070170.

88. Kurtis MM, Rajah T, Delgado LF, Dafsari HS. The effect of deep brain stimulation on the non-motor symptoms of Parkinson's disease: a critical review of the current evidence. NPJ Parkinsons Dis. 2017 Jan;3:16024. DOI 10.1038/npjparkd.2016.24.

89. St George RJ, Carlson-Kuhta P, Burchiel KJ, Hogarth p, Frank N, Horak FB. The effects of subthalamic and pallidal deep brain stimulation on postural responses in patients with Parkinson disease. J Neurosurg. 2012 Jun;116(6):1347-56. DOI 10.3171/2012.2.JNS11847.

90. Odekerken VJ, Boel JA, Schmand BA, de Haan RJ, Figee $M$, van den Munckhof P, et al. GPi vs STN deep brain stimulation for Parkinson disease: Three-year follow-up. Neurology. 2016 Feb;86(8):755-61. DOI 10.1212/WNL.0000000000002401.

91. Odekerken VJ, van Laar T, Staal MJ, Mosch A, Hoffmann CF, Nijssen PC, et al. Subthalamic nucleus versus globus pallidus bilateral deep brain stimulation for advanced Parkinson's disease (NSTAPS study): a randomised controlled trial. Lancet Neurol. 2013 Jan;12(1):37-44. DOI 10.1016/S1474-4422(12)70264-8. 